\title{
Visualisation Techniques for Consent: Finding Common Ground in Comic Art with Indigenous Populations
}

\author{
Maria Wilhelmina Botes \\ IRiSC, Snt \\ University of Luxembourg \\ Esch-sur-Alzette, Luxembourg \\ maria.botes@uni.lu
}

\author{
Arianna Rossi \\ IRiSC, Snt \\ University of Luxembourg \\ Esch-sur-Alzette, Luxembourg \\ arianna.rossi@uni.lu
}

\begin{abstract}
With emerging technologies such as genome research and the digitization of health records comes the need for new models of informed consent. In this climate of innovation people are often prone to explore the latest technological advancement as possible solutions, including for informed consent. In this paper, we present the design and evaluation of a so-called low-tech informed consent solution that was designed specifically for the informational and cultural needs of a vulnerable indigenous population, i.e., the San of South Africa. This low-tech solution took the form of a comic and, although it could enhance understanding and identification, the costs and labour intensity of comic design and the deriving limitations on its scalability should be critically considered in the light of a digitised and more standardized solution.
\end{abstract}

Keywords-informed consent, transparency, icons, comics, genomic research, standardisation, eConsent, indigenous population

\section{INTRODUCTION}

Voluntary informed consent constitutes the foundation of all clinical research without which researchers cannot embark on ethical, legal and valid research projects. Informed consent is defined as autonomous authorisation which relies on the principle of respect for autonomy as self-governance or as the right to self-determination which is the "legal equivalent of the moral principle of the respect for autonomy" and refers to "the right of individuals to make their own decisions without interference from others" [1].

However, the potential for scientific abuse in the absence of informed consent became apparent when many physicians, who have sworn to uphold the Hippocratic Oath and to do no harm failed to respect (what is now well known) basic human rights to justify their inhumane medical experiments [2]. The atrocities committed in the name of scientific progress by the Nazi scientists and medical practitioners during the Second World War are but a single example of such blatant disregard for human rights [3]. When these practices were adjudicated in the well-known Nuremberg Trials, the doctrine of informed consent found its first footing and was further legally embedded in the 1957 case of Salgo v Leland Standford Jr. University Board of Trustees case [4].

In addition to unethical medical experiments committed during war times, the lack of informed consent for further use of the same biomedical samples for other research purposes than initially consented for also negatively impacted the Havasupai tribe in northern America, which caused the tribes to withdraw their sample which led to the termination of the project, thereby limiting further scientific developments [5].

Due to language and educational barriers, scientists also failed to obtain informed consent from the San communities of South Africa, whose genomes have high scientific value because of their diversity and the naturally isolated nature of the population. The publication of sequenced genome data of 4 geriatric San members without their consent in Nature in 2010 [6] led to the deterioration of any form of trust relationship between the San and the scientific community. This situation begged for a novel process of informing illiterate or uneducated research participants and obtaining their consent [7]. This article describes a research study carried out on such consent process, the implications of the findings and its foreseeable evolution.

This article makes the following contributions: (a) it argues why visual forms of consent for biomedial research can better serve the needs of vulnerable, historically exploited communities and better implement its ethical and legal requirements; (b) it shows how such consent comic can enhance understanding and identification of vulnerable communities; (c) it provides an overview of the advantages and disadvantages of customised and standardised consent communication.

This article is organized as follows: Section II illustrates why the San population of South Africa has unique characteristics that represent a valuable asset for scientific research but is also highly vulnerable; Section III introduces ethical and legal requirements to obtain informed consent in such circumstances; Section IV motivates why comics were selected as informed consent tool; Section V details a research study that evaluated whether comprehension and identification are enhanced by comics with respect to textual forms of consent; Section VI shows how the comic consent evolved when the results of the evaluation were taken into account; Section VII discusses the implications of customized comics as a visual consent tool as opposed to standardized visual communication; Section VIII concludes.

\section{VULNERABLE, BUt VALUABLE POPULATIONS}

The San population, whose ancestry dates back more than 100000 years ago, carries the oldest living genes in the world, and subsequently the most diversified [8]. This diversity makes their genes extremely sought after globally for purposes of genomic research to examine genetic variants and traits which will provide important insights into disease development, the spread of diseases and the epigenetic influences that caused such diseases. Such findings can lead to accelerated drug and diagnostic developments [9].

The San population of South Africa is generally poor, unsophisticated, illiterate and not positioned to protect their own interests. Fearing that they may be easily nudged to consent, they are regarded as vulnerable in terms of the Declaration of Helsinki [7]. Although the Declaration of 
Helsinki does not provide a definition for the term, the San population's vulnerability can be deduced from the general ambit of the vulnerability protection provided in the declaration for, amongst others, indigenous populations. In this context the Declaration of Helsinki stipulates that "[s]pecial attention should be given to the specific information needs of individual potential subjects as well as to the methods used to deliver the information" [7]. Accordingly, the San population clearly has special communication needs, because the majority of its members communicate primarily in their indigenous language or in Afrikaans and find it hard, if not impossible, to communicate in English, or any other language, with researchers [10]. Many of its members are also completely illiterate, which questions the legality of any consent form signed in these circumstances.

\section{The Legal MANDATE TO IMPROVE COMMUNICATION}

Most scientific terms associated with genomic research include abstract and complex technical concepts that are often completely foreign to laypeople. Such a hurdle is even more relevant for the members of the San population who suffer from low levels of education and language barriers. Although the San seem to recognize some of the terminology used, it is highly questionable whether they sufficiently comprehend the issues associated with a genome research study to give adequate informed consent as required in international instruments, such as the Belmont Report and the Declaration of Helsinki [10]. The doctrine of informed consent was first introduced into South African law in Richter $v$ Estate Hammann and confirmed in Castell $v$ De Greef as part of the existing South African medical and health law jurisprudence [11]. In terms of the South African Constitution, the bodily and psychological integrity of everyone is protected to the extent that no one may be subjected to medical or scientific experiments without their informed consent [12], and is the informed consent doctrine also codified in the National Health Act [13]. Similarly to the Declaration of Helsinki and the Belmont Report, these acts do not only set out the information that should be disclosed to research participants, but also the need to communicate in a language that the participants understand, taking participants' levels of education, language ability or literacy into account. However, none of the regulatory instruments stipulate how adequate informed consent can or must be obtained to qualify as adequate, especially when such consent must be obtained from a vulnerable population such as the San.

\section{Redesigning Consent: From CaVe Paintings to COMICS}

The San has a very old and rich culture of visual communication to record history or transfer knowledge in the form of cave paintings and rock art, remnants of which can still be seen in the Maluti Mountains and Drakensberg [14]. This visual communication culture seemed to be the ideal common ground for the creation of a comic to explain the concepts associated with genomic research by combining the rock art symbols with graphic icons from the digital world to serve as a visual language to communicate to the San the information required to result in a truly informed consent. This was subsequently the premise on which the visual communication tool depicted in Figure 1 was designed to bridge the communication gaps between a vulnerable indigenous population and genome researchers in $2015^{1}$ [10].

As far as the first author could ascertain, this is the only study of its kind to gauge whether the comprehension of the content of an informed consent instrument can be enhanced in such a naturally isolated vulnerable indigenous population and whether they could identify with the iconic characters that represented the research participants. The further fact that the researcher spoke one of the San's primary languages, namely Afrikaans, also contributed an unanticipated element. Sharing the same language has a positive value on creating a trust relationship with research participants and conveying complex information [10].
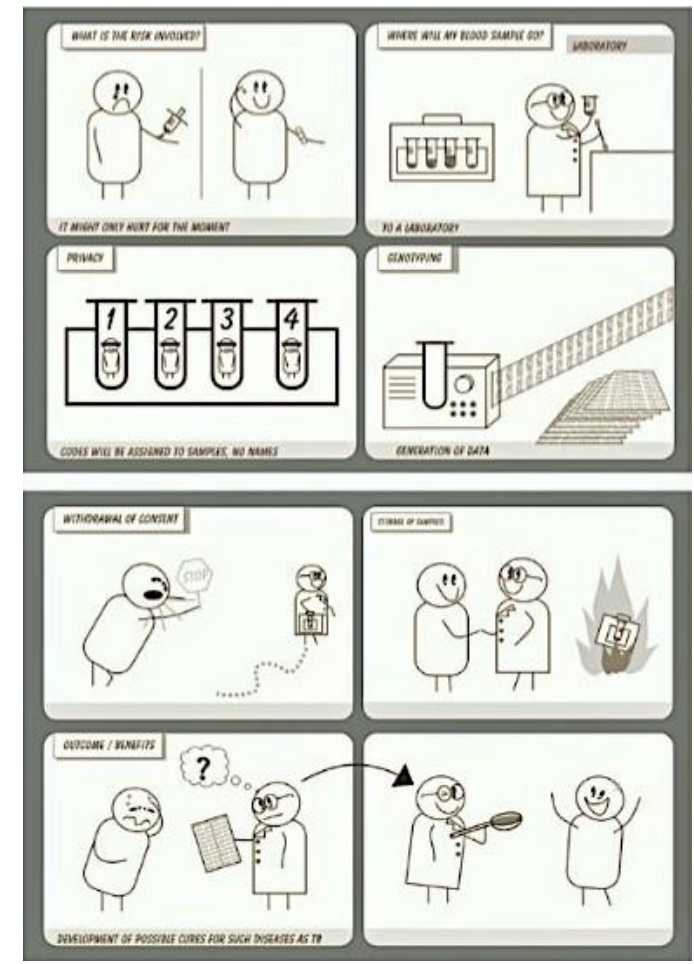

Fig. 1. Pages from the first version of the genome comic that was tested in the San population

\section{Testing Black and White Visuals With Colourful OUTCOMES}

\section{A. Study design}

The entire South African San population consists of 4600 members. 150 members were carefully selected to participate in this study, which is a good proportion of the entire population size. The evaluation of the genome consent comic shown in Figure 1 was carried out in two of the biggest South African San communities consisting of approximately 3500 !Xhun and 1100 Khwe speakers residing at Platfontein, 15 $\mathrm{km}$ from Kimberley in the northern Cape Province. The careful selection of the participants ensured that they were

${ }^{1}$ The study described in this article is a summary of the $\mathrm{PhD}$ thesis research of the first author and is presented in more detail in [10]. 
fairly representative of the age groups of 18-30, 31-60 and 61-80+ years, of various levels of educational development, ability to speak a foreign language also spoken by researchers (e.g., Afrikaans) and gender. The first author followed a qualitative research method to explore the issues of comprehension improvement and identification with the characters, seeking insight rather than a statistical analysis of data. Qualitative, exploratory data was collected by in-depthinterviews and small focus groups with purposefully selected members of the San community [15]. This method was also combined with participatory action research during which the author sought collaboration with members of the South African San council, which are the 'gate keepers' of the San population in South Africa, to determine the population's unique communication challenges, the most appropriate research methods for the involvement of members of their community, and how the results of the analysis of the collected data could be used practically and effectively [16].

The participants were split into two groups with comparable distribution of age, gender, education level and spoken languages. The first group acted as control group and received a traditional written informed consent form, while the second group received the comic consent depicted in Figure 1. Both groups could resort to additional verbal explanations by the researchers, as it is customary in the ethical consent process. Both written and verbal information was provided in Afrikaans. The participants were then asked to answer a set of closed ended questions (i.e., true - false) investigating their level of understanding of the legally required topics illustrated in the consent (e.g., research risks, data sharing, etc.). The participants belonging to the second group could answer some additional open questions meant to explore more general attitudes, for instance about whether the images supported their understanding and whether they could identify with the characters.

\section{B. Study results}

Whereas the group in the control condition could correctly answer $68,30 \%$ (on average) of the questions overall, the group who received the comic showed higher comprehension rate of $76,63 \%$. Thus, the introduction of the genome comic increased participants' comprehension of what genome research entails by $8,33 \%$. Examples of enhanced understanding encompass how individuals inherit genes, how scientists obtain genes from participants, and security measures applied to the gene samples. Many comments also hinted that the participants valued the images for their clarity and their cognitive support to understanding how genome research works.

However, the comic consent also determined a lower comprehension in two of the study questions that dealt with biological terminology and concepts of genome research, namely the methods used for obtaining genetic material (difference of $6,15 \%$ ) and the fact that genes are located inside the human body's cells (difference of 2,89\%). It appears that research participants had preconceived ideas about genes, their location and how scientists extract genes for research purposes. Although some of the research participants previously encountered terminology relating to genes or genome research, it was clear that they held incorrect mental models about these concepts and their actual meaning.
The participants believed that their genes in themselves are a cure for certain diseases, but in fact the evolutionary development of their genes can be used to compare other people's genes during drug investigations and development. Thus, the introduction of the comic appeared to initially confuse some members of the San, because they were forced to revisit such pre-conceived, incorrect ideas when confronted with the correct meaning of genome concepts and terminology.

The only legally orientated question that revealed a less precise comprehension of the participants exposed to the comic content related to data privacy. After the research participants grasped how their genetic samples would be handled and shared, and what kind of information scientists could derive from their biomedical samples, they were concerned. Almost all the participants refused to have their genome information published anywhere (e.g., scientific articles) and were especially concerned about how the San will be depicted and viewed by the international (scientific) community. These concerns arguably stem from the historical exploitation that the San community experienced at the hands of scientists, as discussed earlier, as well as from modern day discrimination and humiliation that they continue to suffer at the hands of local (non-San) communities.

Despite the general increase in genomic understanding, the San had difficulty in identifying with the depicted figures in the comic. The San voiced their desire to see themselves depicted as fully developed characters, dressed in modern western clothing and familiar background surroundings, such as the thorn trees and tall yellow grass that grows in the Northern Cape where they live. The San thought that they were being depicted naked. When the researcher further investigated these perceptions, she found that the icons and avatars used to depict the San was a visible manifestation of how naked the San felt in the eyes of the local South African community as a result of the historical political isolation, degradation and humiliation they have suffered at their hands. This indigenous population had an urgent need to blend into and be acknowledged by the local South African communities as one of their own, hence the requirement for the redesign of the visual communication tool so that they could be depicted as looking like one of 'them' and not as a tourist attraction.

\section{BACK TO THE DRAWING BOARD}

The San's need to be depicted in a more realistic way, in full colour and as a fully developed and clothed human character confirms the theory by cartoonist Scott McCloud that those who prefer a more realistic depiction of visuals tend to have a more concrete perception of their environment, as opposed to the abstraction of symbols used in the written word [17]. Many of the San people also requested the use of only pictures and no words, which underscore their general concrete perception of their environment and tendency to move away from the abstraction of the written word to gravitate more towards concrete depictions. The participants with a higher level of education favoured the use of words in the comic more, whilst participants with lower or no levels of education favoured the exclusive use of pictures in the comic. 
Following the above discussed research outcomes, the author redesigned the genome consent comic as depicted in Figure 2. This version boasts full colour panels with much more background detail, as requested by the San during the study. Characters have also been developed into detailed characters and depict the San and scientists as fully clothed in modern day fashions and with smiling faces, as explicitly requested by the San. Text in this comic has been kept to the minimum but was still included to support the interpretation of the meaning of the images.

Besides being entertaining and engaging, comics (including simple line sketches like the first consent version) have a way of conveying ideas that words or even photo realism cannot do. By abstracting an image through cartooning into a comic, many details from realistic images are eliminated to the extent that one can focus on the most important aspects.[25] Comic art allows for the stripping down of any concept into their essential meaning, which can further be amplified to ensure proper understanding of such concept, which is incomparable to other means of communication like text.

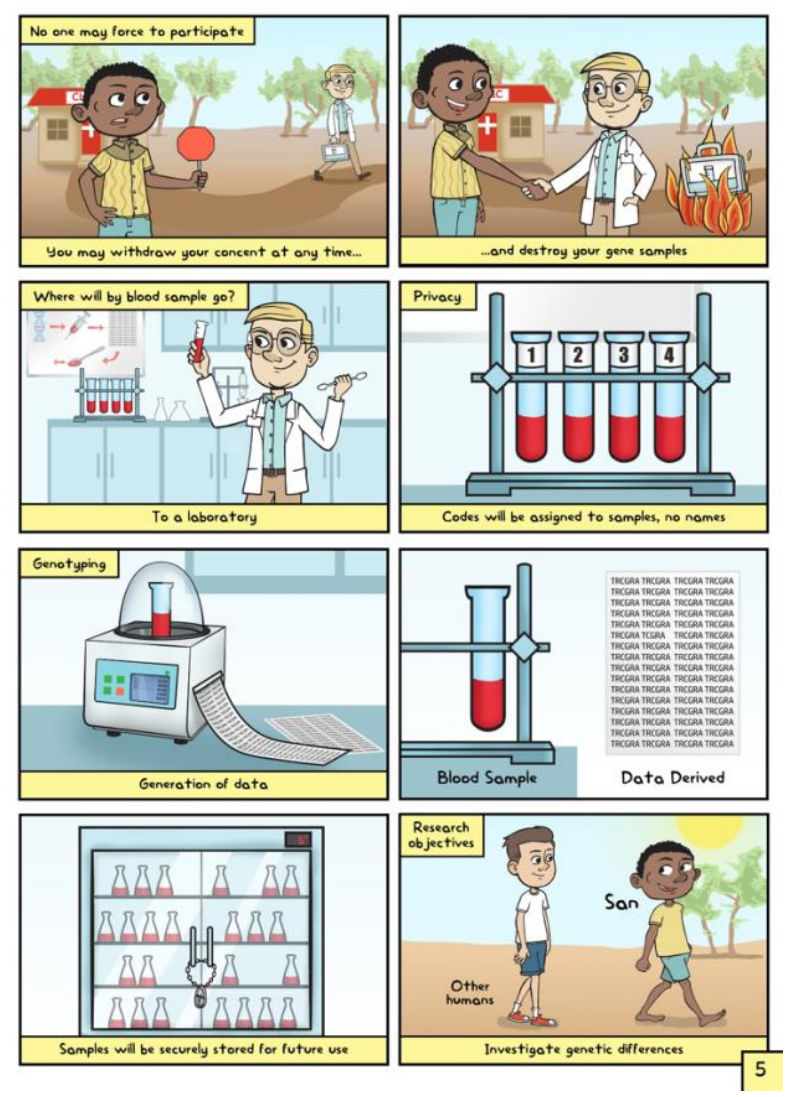

Fig. 2. Pages from the redesigned consent comic in accordance with the research outcomes

By characterising science through comics, abstract concepts such as DNA, viruses or gene editing may be made visible to people with a limited understanding of these scientific concepts. They can transform the reader into an active participant of the information acquiring process and provide them with more agency and autonomy when making important decisions [26]. In the case of the San community, their personally designed comic also gave them a sense of dignity by being acknowledged in a way they have always longed for, thereby re-establishing some of the trust previously lost through historical exploitation.

\section{VISUAL STANDARDS FOR TRANSPARENCY AND CONSENT}

Unfortunately, high-quality comics are notoriously expensive and labour intensive to produce. In line with the move towards digitisation, more standardized formats of consent designs provide a cheaper alternative, especially if the consent tool needs to be scaled to thousands and even millions of individuals. This is why a standardised visual language for transparently conveying the complex notions necessary for consent to be informed should be established.

A comparison can be made with the recent efforts made in the data protection domain to enhance the comprehensibility of communication about privacy risks and data processing practices, with a focus on the European Union. The General Data Protection Regulation [18] has established clear transparency requirements concerning what information must be disclosed and how it must be presented to individuals for consent to be informed. Such requirements mandate the exact information items about personal data processing that should be communicated (Art. 13), like the purposes of data collection and the rights that data subjects can exercise. Moreover, they also give straightforward indications about how information should be provided, namely in a concise manner and in clear and plain language. Such requirements echo the importance of the accessibility of language to ensure comprehension that has been also acknowledged by the South African acts, the Declaration of Helsinki and the Belmont Report. But additionally, the GDPR introduces the idea of using standardised machine-readable icons in combination with text to enhance its visibility and intelligibility (Art 12). Moreover, it sets out clearly the characteristics that consent should have to be considered legally valid: be freely given, specific, informed and unambiguous (Art. 4). When it lifts the prohibition of processing sensitive data like genomic data, it should additionally be explicit.

The establishment of clear user-centric requirements for information disclosures and informed consent is a crucial first step towards the standardization of communication which, among the others, would have the benefit of presenting information homogeneously across products, services and nations. Standardization of communication could allegedly improve information comparison and understanding about important privacy aspects. By also standardizing how consent choices shall be provided to individuals, higher levels of protection may be achieved, as individuals would already be familiar with previously encountered consent mechanisms and hence could make better informed choices. The ISO standard on privacy notices and consent [19] contains similar requirements [20] and mentions textual, graphical and visual formats for information disclosure, including icons.

The quest for a standard visual lexicon to express data privacy concepts is long-standing, but still very active in the 
$\mathrm{EU}^{2}$ and in the US [21]. The supposed benefits are many [22]. First, a visual code can summarise the main data practices of a service and be combined or even replace lengthy text-heavy disclosures that are off-putting by default. Second, visuals offer the advantage of capturing and retaining attention more easily than text. Third, images can cross language boundaries and literacy levels and thus act as a universally comprehensible language - which is the idea that originated the comic consent presented earlier. Moreover, a simplified visual alphabet, for instance composed of pictograms or labels, would be easy to scale and to reproduce on different formats, both digital and not. Since icons would provide short visual summaries of information that is often presented as complex, lengthy and impenetrable text and would even highlight the relevant information to make consent decisions, they can be rightfully considered a low-tech visualization aid that can, in principle, enhance transparency and support the right to autonomy and self-determination. A standardized icon language can be useful beyond the domain of data privacy to simply convey biomedical research concept options, similarly to the symbols that already act as wayfinding systems in many hospitals [23]. Its effectiveness to help the taking of informed decision about data sharing should be further researched for implementation into the standardized consent form for data altruism foreseen by the draft version of the Data Governance Act [24]. Clarity of information and choices could enable greater data portability and thereby support the development of new services for the common good.

That said, boiling down the complexity of legal or technical concepts to a graphical symbol signifies that many nuances of the meaning will be simplified and even lost in such translation. Languages have an infinite number of ways of combining their many words and they are therefore infinitively creative. They can express any meaning, even if it was never expressed before, with the greatest precision - and this is why humans have invented technical jargon. However, with standardized visual languages made of simple pictograms, precision of meaning may be sacrificed for the sake of summarizing the main concepts and democratizing their comprehensibility. For instance, the symbols for men with trousers and women with a dress do not faithfully represent reality, but they are nevertheless accepted and understood internationally. Similarly, labels for privacy ${ }^{3}$, as well as for food and under products, may reveal their level of quality at a glance, but do not reveal neither which criteria were selected and considered to design the label, nor how the grades were calculated. Yet, labels are arguably a better decision-making tool for unexpert individuals that would never delve into the complexity of specialistic information and even if they did, they would hardly grasp its explicit and implied meanings. Such complexity is undoubtedly lost to the end-user and with it the underlying motivations that brought to the final simplified design. It could be argued that comics and other forms of visual language also simplify the reality and nudge the reader to focus on certain aspects over others. However, enlarging the comprehension rates of wider numbers of population with respect to risky privacy practices and sensitive biomedical research, even at the expenses of precision of meaning, may be easily considered a better trade off than achieving only limited comprehension.
Moreover, despite enhanced visual saliency, the comprehensibility of depicted information does not necessarily augment, especially when using icons. The meaning of unfamiliar or abstract visuals shall be learnt, thus greater attention should be paid to the integration with textual descriptors, as it was achieved in the comic consent version. When it not implied realistic representations like comics, a visual lexicon can become a universal language only provided that a specific combination of symbols (and forms, and colors) has been identified and standardised via international conventions (e.g., traffic signs, safety signs, etc.), regulations (e.g., energy consumption labels in the EU) or widespread and uniform use (e.g., the padlock to signify secure connection adopted by all browsers) [25]. This is exactly why the symbols for men and women can be found in many countries and do not originate misunderstandings.

Lastly, standard graphical symbols are not always the most appropriate solutions to better communicate consent choices to any population. The idea of using icons in the GDPR is based on the assumption that the European population is quite uniform, for instance concerning their digital skills and thereby their ability to navigate privacy choices. However, studies show that the digital divide in the EU exists and derives mostly from differences in age, educational level, and occupation [26]. The study on the San population suggests that certain communities feel the need for identification with more realistic characters depicted in more detailed environments, as opposed to standardized representations.

\section{CONCLUSIONS}

Since its formal introduction as one of the rules handed down during the Nuremberg trials in 1945, informed consent has been created and implemented as a written document, with a focus on the exhaustiveness of its content. Only recently, other methods to convey the content of informed consent forms and processes such as comics have been developed. But considering that genome studies often involved large populations, the scaling of personalised or culture-specific comics seems to be a luxury if the developmental costs and scalability thereof is considered. For this reason, standardization initiatives regarding consent, including privacy icons, have been gaining momentum. Moreover, given the increased digitisation of data, consent models and platforms are also being transformed into electronicallymediated processes. Like comics, e-consent also harbours the possibility of providing an engaging and informative consent experience through the use of interactive digitised interfaces.

Current developments in consent and information dissemination open promising possibilities to merge models, theories, media and methods to provide innovative and informative informed consent experiences. In the future, modularity may help to design consent experiences that will consist of a combination of icons, comics, audiobooks, virtual reality tools, engaging interfaces and more, subject to laudable standardisation efforts based on meaningful, customisable consent options to leverage the opportunities of the data economy. 


\section{ACKNOWLEDGMENT}

This work has been partially supported by the Luxembourg National Research Fund (FNR) IS/14717072 "Decepticon" and IS/14638785 "ConGenIAl". This research would have been impossible without the support from the San Council of South Africa and the research participants. We would finally like to thank the anonymous reviewers for their useful comments.

\section{REFERENCES}

[1] R. R. Faden and T. L. Beauchamp, A history and theory of informed consent. Oxford University Press, 1986.

[2] S. H. Miles, The Hippocratic Oath and the ethics of medicine. Oxford University Press, 2005.

[3] B. Halioua, "The Participation of Hans Hinselmann in Medical Experiments at Auschwitz," J. Low. Genit. Tract Dis., vol. 14, no. 1, pp. 1-4, Jan. 2010, doi: 10.1097/LGT.0b013e3181af30ef.

[4] Salgo v. Leland Stanford etc. Bd. Trustees. 1957.

[5] N. Garrison, "Genomic justice for Native Americans: impact of the Havasupai case on genetic research," Sci. Technol. Hum. Values, vol. 38, no. 2, pp. 201-223, 2013.

[6] S. C. Schuster et al., "Complete Khoisan and Bantu genomes from southern Africa," Nature, vol. 463, no. 7283, pp. 943-947, Feb. 2010, doi: 10.1038 /nature08795.

[7] "World Medical Association Declaration of Helsinki: Ethical Principles for Medical Research Involving Human Subjects," JAMA, vol. 310, no. 20, p. 2191, Nov. 2013, doi: 10.1001/jama.2013.281053.

[8] C. M. Schlebusch et al., "Genomic variation in seven Khoe-San groups reveals adaptation and complex African history," Science, vol. 338, no. 6105, pp. 374 379, 2012.

[9] E. Cano-Gamez and G. Trynka, "From GWAS to function: using functional genomics to identify the mechanisms underlying complex diseases," Front. Genet., vol. 11, p. 424, 2020.

[10] W. M. B. Steenberg, "Visual communication as a legalethical tool for informed consent in genome research involving the San community of South Africa Visual communication as a legal-ethical tool for informed consent in genome research involving the San community of South Africa," PhD Thesis, University of South Africa, 2017.

[11] “Castell v De Greef," Afr Law Rep, vol. 4, pp. 408-41, 1994.

[12] "Section 12," in Constitution of the Republic of South Africa, 1996.

[13] National Health Act. 2003.

[14] D. Lewis-Williams, D. M. Witelson, D. Pearce, and S. Challis, "An ancient San rock art mural in South Africa reveals new meaning," The Conversation. http://theconversation.com/an-ancient-san-rock-artmural-in-south-africa-reveals-new-meaning-157177 (accessed Jul. 16, 2021).
[15] J. M. Morse and P. A. Field, Nursing research: The application of qualitative approaches. Nelson Thornes, 1995.

[16] H. S. Speziale, H. J. Streubert, and D. R. Carpenter, Qualitative research in nursing: Advancing the humanistic imperative. Lippincott Williams \& Wilkins, 2011.

[17] S. McCloud, "Understanding comics: The invisible art," Northamp. Mass, 1993.

[18] European Parliament and Council of European Union, Regulation (EU) 2016/679 of the European Parliament and of the Council of 27 April 2016 on the protection of natural persons with regard to the processing of personal data and on the free movement of such data, and repealing Directive 95/46/EC (General Data Protection Regulation). 2016.

[19] ISO, "ISO/IEC 29184:2020. Information technology - Online privacy notices and consent." 2020. Accessed: Jun. 10, 2021. [Online]. Available: https://www.iso.org/cms/render/live/en/sites/isoorg/co ntents/data/standard/07/03/70331.html

[20] H. Pandit and G. P. Krog, "Comparison of notice requirements for consent between ISO/IEC 29184:2020 and GDPR," J. Data Prot. Priv., vol. 4, no. 2, pp. 193-204, 2021.

[21] H. Habib et al., "Toggles, Dollar Signs, and Triangles: How to (In)Effectively Convey Privacy Choices with Icons and Link Texts," in Proceedings of the 2021 CHI Conference on Human Factors in Computing Systems, Yokohama Japan, May 2021, pp. 1-25. doi: 10.1145/3411764.3445387.

[22] A. Rossi and M. Palmirani, "What's in an Icon? Promises and Pitfalls of Data Protection Iconography," in Data Protection and Privacy: Data Protection and Democracy, S. G. Ronald Leenes Dara Hallinan and P. D. Hert, Eds. Hart publishing, 2020, pp. 59-92.

[23] D. R. Vaillancourt, D. G. Villarreal, and D. A. Pouliot, "Pictograms," Visible Lang., p. 13.

[24] European Commission, Proposal for a REGULATION OF THE EUROPEAN PARLIAMENT AND OF THE COUNCIL on European data governance (Data Governance Act). COM(2020) 767 final 2020/0340 (COD). 2020. Accessed: Jul. 16, 2021. [Online]. Available: $\quad$ https://eur-lex.europa.eu/legalcontent/EN/TXT/PDF/?uri=CELEX:52020PC0767\&f rom $=\mathrm{EN}$

[25] A. Rossi and G. Lenzini, "Making the Case for Evidence-based Standardization of Data Privacy and Data Protection Visual Indicators," J. Open Access Law JOAL, vol. 8, no. 1, 2020.

[26] M. D. Vasilescu, A. C. Serban, G. C. Dimian, M. I. Aceleanu, and X. Picatoste, "Digital divide, skills and perceptions on digitalisation in the European UnionTowards a smart labour market," PLoS ONE, vol. 15, no. 4, p. e0232032, Apr. 2020, doi: 10.1371/journal.pone.0232032. 